\title{
Superrotation in Terrestrial Atmospheres
}

\author{
ANNE L. LARAIA \\ California Institute of Technology, Pasadena, California \\ TAPIO SCHNEIDER \\ ETH Zürich, Zurich, Switzerland, and California Institute of Technology, Pasadena, California
}

(Manuscript received 22 January 2015, in final form 2 July 2015)

\begin{abstract}
Atmospheric superrotation with prograde equatorial winds and an equatorial angular momentum maximum is ubiquitous in planetary atmospheres. It is clear that eddy fluxes of angular momentum toward the equator are necessary to generate it. But under what conditions superrotation arises has remained unclear. This paper presents simulations and a scaling theory that establish conditions under which superrotation occurs in terrestrial atmospheres. Whether superrotation arises depends on the relative importance of factors that favor or disfavor superrotation. Convection preferentially generates Rossby waves near the equator, where the Rossby number is $O(1)$. Since the Rossby waves transport angular momentum toward their source regions, this favors superrotation. Meridional temperature gradients preferentially lead to baroclinic instability and wave generation away from the equator. Eddy transport of angular momentum toward the baroclinic source region implies transport out of low latitudes, which disfavors superrotation. Simulations with an idealized GCM show that superrotation tends to arise when the equatorial convective generation of wave activity and its associated eddy angular momentum flux convergence exceed the baroclinic eddy angular momentum flux divergence. Convective and baroclinic wave activity generation is related through scaling arguments to mean-flow properties, such as planetary rotation rates and meridional temperature gradients. The scaling arguments show, for example, that superrotation is favored when the off-equatorial baroclinicity and planetary rotation rates are low, as they are, for example, on Venus. Similarly, superrotation is favored when the convective heating strengthens, which may account for the superrotation seen in extreme global warming simulations.
\end{abstract}

\section{Introduction}

Atmospheric superrotation refers to a local angular momentum maximum in the fluid interior. Because angular momentum must decrease toward the poles for the flow to be inertially stable, atmospheric superrotation usually means equatorial superrotation: that is, a local angular momentum maximum at the equator (Held 1999). Such atmospheric superrotation may be the norm rather than the exception. Venus and Titan have superrotating atmospheres (Schubert 1983; Gierasch et al. 1997; Kostiuk et al. 2001). Jupiter's and Saturn's atmospheres also superrotate (Porco et al. 2003; SánchezLavega et al. 2007), but because they do not have a solid

Corresponding author address: Tapio Schneider, ETH Zürich, Sonneggstr. 5, 8092 Zurich, Switzerland.

E-mail: tapio@ethz.ch surface, they superrotate relative to the rotation of their cores and magnetic fields. For an atmosphere to superrotate, it needs to have angular momentum fluxes into the region of superrotation (Hide 1969). Inviscid axisymmetric circulations cannot accomplish this upgradient angular momentum transport; eddies must be involved (Held and Hou 1980; Schneider 2006). In general, in sufficiently rapidly rotating atmospheres, eddy angular momentum fluxes converge into the regions in which wave activity is generated, and they diverge where wave activity is dissipated (Held 1975; Andrews and Mcintyre 1976; Edmon et al. 1980). Thus, preferential wave activity generation near the equator is a prerequisite for superrotation. This must not be overcompensated by wave activity dissipation near the equator, for example, associated with baroclinic eddies that are generated in midlatitudes and dissipate preferentially in lower latitudes, as they do on Earth (Saravanan 1993). 
Various mechanisms are available for preferential wave activity generation near the equator. A stationary heat source near the equator, for example, leads to the generation of stationary Rossby waves, which can dissipate away from the equator and so transport angular momentum toward the equator. This leads to superrotation when the heat source is strong enough (Suarez and Duffy 1992; Saravanan 1993; Kraucunas and Hartmann 2005; Arnold et al. 2012). The stationary wave mechanism is responsible for superrotation in simulations of tidally locked planets, in which stellar heating is radially symmetric around an equatorial focal point (Joshi et al. 1997; Merlis and Schneider 2010; Pierrehumbert 2011). For a planet without deviations from axisymmetry in boundary conditions, it is less obvious why waves should be preferentially generated near the equator. Wang and Mitchell (2014) and Pinto and Mitchell (2014) find that a Rossby-Kelvin instability produces angular momentum flux convergence at the equator that is responsible for the generation of superrotation in statically stable atmospheres. In convecting atmospheres, the variation of the Rossby number with latitude provides an alternative mechanism: Near the equator, where the Rossby number can be $O(1)$, horizontal and temporal temperature variations are small when the Froude number is small. Therefore, fluctuations in convective heating must be balanced by vertical motion and hence by horizontal divergence at the level of the convective outflows in the upper troposphere (Charney 1963; Sobel et al. 2001). The horizontal divergence then can generate large-scale rotational flow and thereby Rossby waves, either by vortex stretching or vorticity advection (Sardeshmukh and Hoskins 1988). In contrast, in higher latitudes, where the Rossby number is small, convective heating fluctuations can, for example, be balanced by transient temperature fluctuations, which may relax radiatively without generating largescale waves that dissipate in other latitude bands. The net result is preferential generation of Rossby waves near the equator by convective heating fluctuations. If some of these convectively generated Rossby waves dissipate at higher latitudes-for example, through interaction with the mean-flow shear-they will transport angular momentum toward the equator and thus can generate superrotation (Schneider and Liu 2009; Liu and Schneider 2010).

However, angular momentum flux convergence associated with preferential wave activity generation at the equator may be counterbalanced or overcompensated by angular momentum flux divergence associated with dissipation of wave activity that was generated at higher latitudes (e.g., by baroclinic instability) (Saravanan 1993). This is the case in Earth's troposphere in the annual mean, and it may be the case on Uranus and Neptune, which are subrotating (Liu and Schneider 2010). Only when the baroclinically unstable region is moved into low latitudes by artificially increasing radiative heating gradients near the equator and reducing them in higher latitudes can baroclinic instability promote the onset of superrotation (Williams 2003).

Here, we focus on equatorial superrotation on terrestrial planets: that is, planets with solid surfaces with a distribution of radiative heating rates resembling Earth's. We explore a wide parameter regime that encompasses subrotating (Earth like) and superrotating atmospheres. Our goal is to elucidate the mechanisms that generate and maintain tropospheric superrotation in convecting atmospheres and quantify the conditions under which superrotation generally arises. ${ }^{1} \mathrm{We}$ quantify the relative importance of the angular momentum fluxes associated with equatorial convectively generated waves and off-equatorial baroclinic eddies. We use simulations with an idealized GCM to demonstrate that whether superrotation occurs in terrestrial atmospheres depends on the competition between the two, and we use scaling arguments to estimate their relative importance in terms of mean-flow quantities and external parameters.

\section{Idealized GCM and simulations}

The idealized GCM used for the simulations is based on the dynamical core of the Geophysical Fluid Dynamics Laboratory's Flexible Modeling System. It performs a time integration of the primitive equations of motion on a sphere with Earth's radius, using the spectral transform method in the horizontal and using 30 $\sigma$ levels in the vertical. Here, $\sigma=p / p_{s}$ is a dimensionless vertical coordinate, where $p$ is the pressure and $p_{s}$ is the surface pressure; it ranges from 1 at the surface to 0 at the top of the atmosphere. All simulations were performed at T85 horizontal resolution.

Neither seasonal nor diurnal cycles of insolation are included in this model, and there is no topography. The GCM treats the atmosphere as an ideal gas without a hydrologic cycle. The effects of moisture are generally ignored, but they are implicit in a convection parameterization, which relaxes atmospheric temperatures to a profile with lapse rate equal to a fraction $\gamma \leq 1$ of the dry adiabatic lapse rate $\Gamma_{d}=g / c_{p} \approx 9.8 \mathrm{~K} \mathrm{~km}^{-1}$.

\footnotetext{
${ }^{1}$ Superrotation can also occur in the stratosphere, for example, during the westerly phase of the quasi-biennial oscillation. The mechanisms responsible for that are different from those in the troposphere, which is our focus here.
} 
Whenever an atmospheric column is less stable than the specified convective lapse rate $\gamma \Gamma_{d}$, a dry convection scheme relaxes temperatures to a profile with the convective lapse rate, while conserving the columnintegrated enthalpy (Schneider and Walker 2006). That is, the convection scheme assumes that the kinetic energy of the convection is locally dissipated on the convective (subgrid) scale so that the enthalpy on the large (grid) scale is conserved. The rescaling factor $\gamma$ mimics the effect of latent heat release in moist convection, with smaller $\gamma$ corresponding to more latent heat release.

A Newtonian relaxation scheme represents radiative forcing by relaxing temperatures toward radiativeequilibrium temperatures $T_{e}$. Unlike the usual statically stable equilibrium temperature fields used in many idealized dry GCMs, these radiative-equilibrium temperatures are statically unstable in the lower and middle troposphere. The radiative-equilibrium surface temperature in the model is a function of latitude $\phi$ and is given by

$$
T_{s}^{e}(\phi)=\tilde{T}_{s}^{e}+\Delta_{h} \cos ^{2} \phi
$$

The radiative-equilibrium surface temperature at the pole is fixed at $\tilde{T}_{s}^{e}=260 \mathrm{~K}$ in all simulations. Radiativeequilibrium temperatures in the atmosphere above the surface are those of a semigray atmosphere with a water vapor-like absorber. Their meridional gradient smoothly decays to zero at the top of the atmosphere, where the radiative-equilibrium temperature is a constant 200 K. See Schneider (2004) for more details on the Newtonian relaxation scheme and Schneider and Walker (2006) for a more detailed model description. The only difference between our model and that described in Schneider and Walker (2006) is that we represent subgrid-scale dissipation by an exponential cutoff filter (Smith et al. 2002), with a damping time scale of 4 min on the smallest resolved scale and a cutoff wavenumber of 21 below which subgrid-scale dissipation is zero. Weaker subgrid-scale damping would have sufficed for many simulations but led to substantial noise in some superrotating simulations, especially the ones with weak meridional temperature gradients and more stable convective lapse rates. Thus, we use the stronger damping for all simulations to be consistent. We also found the magnitude of the superrotating wind speed to be quite sensitive to the damping time scale of the subgrid-scale dissipation.

We performed 60 simulations by varying three model parameters: the pole-equator temperature contrast in radiative equilibrium $\Delta_{h}$, the planetary rotation rate $\Omega$, and the rescaling parameter $\gamma$ in the convective lapse
TABLE 1. Parameters varied in the 60 simulations: planetary rotation rate $\Omega$ (as a multiple of Earth's rotation rate $\Omega_{e}$ ), poleequator temperature contrast in radiative equilibrium $\Delta_{h}$, and rescaling parameter $\gamma$ of the convective lapse rate $\Gamma=\gamma \Gamma_{d}$.

\begin{tabular}{cl}
\hline \hline Parameter & \multicolumn{1}{c}{ Values } \\
\hline$\Omega$ & $\left(2,1,2^{-1}, 2^{-2}, 2^{-3}\right) \times \Omega_{e}$ \\
$\Delta_{h}$ & $30,60,120,240 \mathrm{~K}$ \\
$\gamma$ & $0.5,0.7,0.9$ \\
\hline
\end{tabular}

rate $\gamma \Gamma_{d}$. Table 1 shows the different parameter values used to create a range of different climate scenarios. We chose to vary these parameters because, based on previous work (e.g., Del Genio et al. 1993; Del Genio and Zhou 1996; Liu and Schneider 2011; Mitchell and Vallis 2010; Potter et al. 2014; Pinto and Mitchell 2014), they seemed to be pertinent to the generation of superrotation. Because rescaling the planetary rotation rate in a hydrostatic model like ours is dynamically equivalent to rescaling both the planetary radius and diabatic heating rates (e.g., Kuang et al. 2005; Garner et al. 2007), we did not consider separate variations of the planetary radius. However, variations of diabatic time scales would be interesting to explore systematically in a future study (cf. Yamamoto and Takahashi 2006; Pinto and Mitchell 2014).

All simulations were integrated for at least 1500 days, and the model output is averaged over the last 400 days of each simulation.

\section{Results}

\section{a. Circulation variations}

Our goal is to determine why some simulations superrotate and some do not. Figure 1 displays two superrotating and two subrotating simulations. The left column shows the eddy angular momentum flux divergence (colors) and zonal wind (black contours), and the right column shows the mass flux streamfunction, with solid lines for counterclockwise rotation and dotted lines for clockwise rotation. The top row shows an Earthlike reference simulation, with Earth's rotation rate $\Omega=\Omega_{e}$, a convective lapse rate of $\Gamma \approx 6.9 \mathrm{~K} \mathrm{~km}^{-1}$ $(\gamma=0.7)$, and pole-equator radiative-equilibrium temperature contrast of $\Delta_{h}=120 \mathrm{~K}$. As on Earth, there are two westerly jets located in the upper troposphere in midlatitudes, with weak easterlies at the equator and poles. In the right column, we can see the Hadley circulation in the tropics and the Ferrel cells in midlatitudes.

The simulation in the second row has the same Earthlike parameter values for convective lapse rate and pole-equator temperature contrast but a planetary 

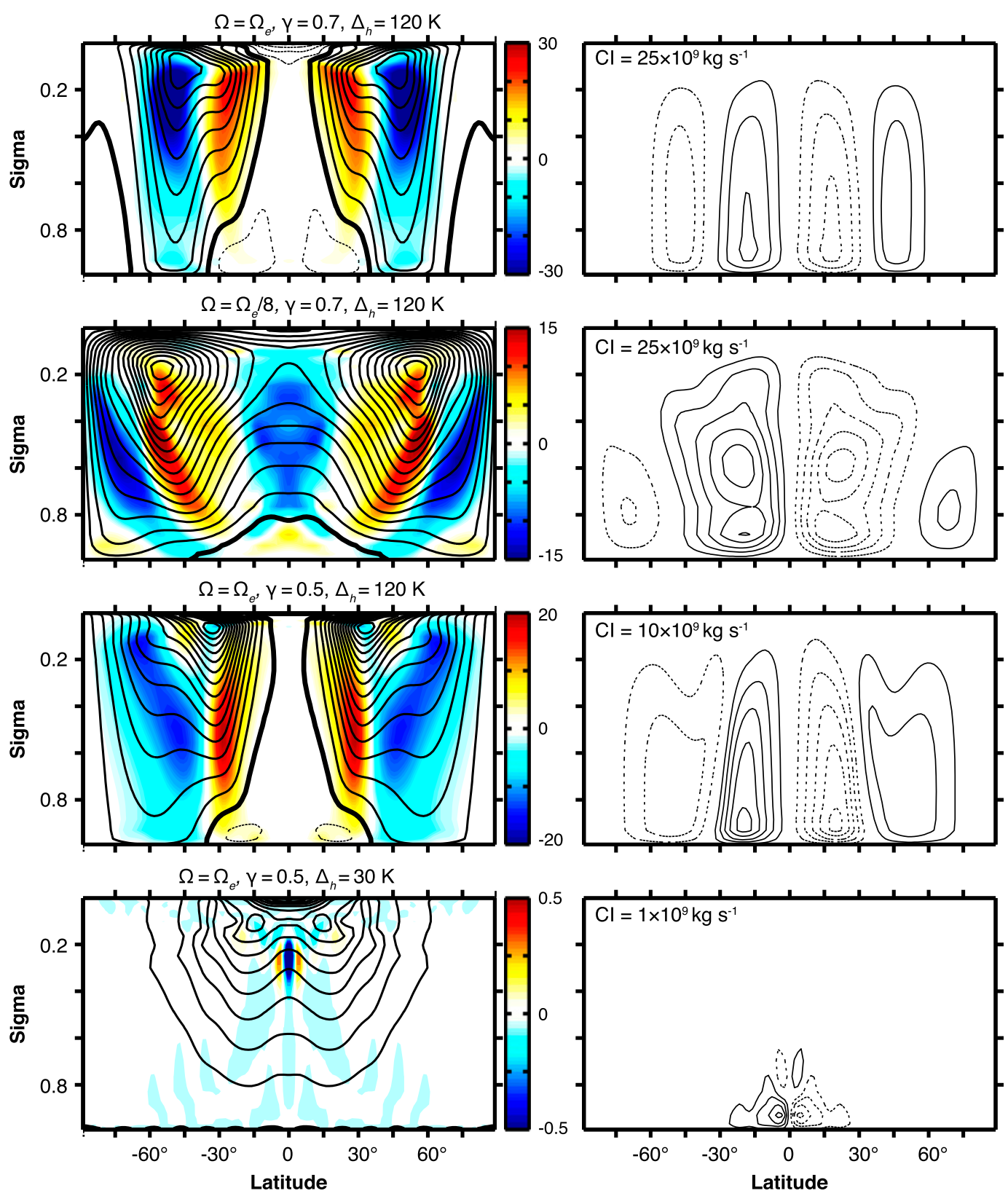

FIG. 1. (left) Zonal-mean zonal wind (black contours; contour interval: $5 \mathrm{~m} \mathrm{~s}^{-1}$ ) and eddy angular momentum flux divergence (colors; $10^{-6} \mathrm{~m} \mathrm{~s}^{-2}$ ) in the latitude-sigma plane. The thick black line is the zero zonal-wind contour. (right) Eulerian-mean mass flux streamfunction, with contour intervals given in each panel. Four simulations are shown, with their parameter values indicated in the left column.

rotation rate $\Omega=\Omega_{e} / 8$. The westerly jets are situated farther poleward, and the surface easterlies at the equator are very weak. The equatorial upper troposphere is strongly superrotating, with strong eddy angular momentum flux convergence in the superrotating region. The Hadley cells have expanded poleward as well, as is to be expected for lower planetary rotation rates (Walker and Schneider 2006).
The simulation in the third row is one with Earth's parameter values, except for a smaller, more stable convective lapse rate $\Gamma \approx 4.9 \mathrm{~K} \mathrm{~km}^{-1}(\gamma=0.5)$. The Hadley cells weaken (note the difference in contour interval among the different simulations) because the reduced baroclinicity implies reduced eddy angular momentum flux divergence in the subtropics (Walker and Schneider 2006; Schneider and Walker 2008). 


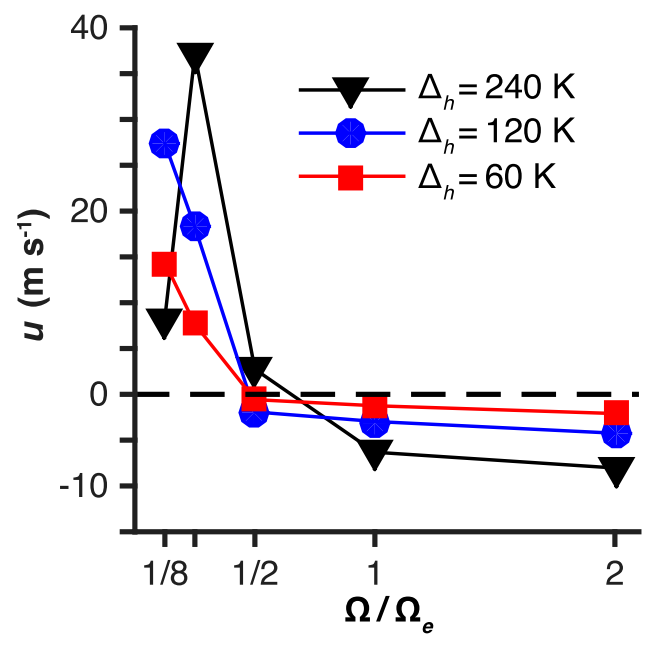

FIG. 2. Upper-tropospheric zonal wind at the equator vs $\Omega / \Omega_{e}$, with fixed values of $\Delta_{h}$ and $\gamma$. For all three curves, $\gamma=0.7$.

Additionally, the zonal jets extend farther equatorward than in the reference simulation in the top row.

The simulation in the bottom row differs from the simulation above it only in the reduced pole-equator radiative-equilibrium temperature contrast of $\Delta_{h}=30 \mathrm{~K}$. Decreasing the baroclinicity of the atmosphere produces a superrotating atmosphere with a much weaker meridional circulation.

All other parameters held constant, a decrease in the planetary rotation rate generally leads to an increase in the average equatorial wind speed (Fig. 2). There are deviations from this behavior that occur at the lowest rotation rates, when the midlatitude westerly jets migrate toward the poles, as was already seen in simulations by Del Genio and Zhou (1996).

For faster planetary rotation rates, the equatorial winds become more westerly with decreasing $\Delta_{h}$ (cf. third and fourth rows in Fig. 1). This is in line with the notion that larger meridional temperature gradients produce stronger baroclinic instability in midlatitudes, leading to offequatorial wave generation and angular momentum flux divergence near the equator. For slower rotation rates, however, the opposite is true. In these cases, the atmospheres are superrotating at every value of $\Delta_{h}$ we explored, and the superrotation becomes more pronounced for larger $\Delta_{h}$ (Fig. 2). So, for a subrotating atmosphere, reducing the meridional temperature gradient can eventually lead to superrotation. On the other hand, decreasing the meridional temperature gradient when the atmosphere is strongly superrotating (e.g., for a slowly rotating planet) can weaken the superrotation.

\section{b. Relation to wave activity sources}

Why do some simulations superrotate while others do not? Quantifying the amount of equatorial eddy angular momentum flux divergence and convergence owing to convective and baroclinic sources provides insight. As stated above, there must be a wave activity source at the equator that produces eddy angular momentum fluxes directed into the equatorial region in order to generate and sustain the angular momentum maximum there. Making the weak temperature gradient approximation near the equator, we write the vorticity equation neglecting the baroclinic term, the twisting-tilting term, and friction:

$$
\frac{\partial \zeta_{a}}{\partial t}+\mathbf{v}_{\Psi} \cdot \nabla_{h} \zeta_{a} \approx-\zeta_{a}\left(\nabla_{h} \cdot \mathbf{v}_{\chi}\right)-\left(\mathbf{v}_{\chi} \cdot \nabla_{h} \zeta_{a}\right)=R
$$

Here, $\zeta_{a}$ is the absolute vorticity, $\mathbf{v}_{\chi}+\mathbf{v}_{\Psi}=\mathbf{v}$ is the horizontal velocity decomposed into its rotational $(\Psi)$ and divergent $(\chi)$ components, and $\nabla_{h}$ denotes the horizontal components of the del operator. Following Sardeshmukh and Hoskins (1988) and Schneider and Liu (2009), we define the Rossby wave source as the fluctuating part of the right-hand side of this equation, $R^{\prime}=R-\bar{R}$, with the overbar denoting a zonal mean and primes denoting deviations therefrom.

The vorticity equation implies the approximate eddy enstrophy equation:.

$$
\frac{1}{2} \frac{\partial \overline{\zeta^{\prime 2}}}{\partial t}+\overline{v_{\Psi}^{\prime} \zeta^{\prime}} \partial_{y} \bar{\zeta}_{a} \approx \overline{R^{\prime} \zeta^{\prime}}
$$

where $v_{\Psi}$ is the rotational component of the meridional velocity $v$, and $y=a \phi$ is the meridional coordinate, with planetary radius $a$. Advection of eddy vorticity by the eddies themselves (the triple correlation term) is neglected. In a statistically steady state and under the assumption that $\bar{\zeta}_{a} \neq 0$, the wave activity balance at the equator can then be written as (Andrews and Mcintyre 1976; Andrews and McIntyre 1978; Edmon et al. 1980; Schneider and Liu 2009)

$$
G=\frac{\overline{R^{\prime} \zeta^{\prime}}}{\partial_{y} \bar{\zeta}_{a}}=\overline{v_{\Psi}^{\prime} \zeta^{\prime}}=-\frac{1}{\cos \phi} \operatorname{div}\left(\overline{u_{\Psi}^{\prime} v_{\Psi}^{\prime}} \cos \phi\right)
$$

where div is the meridional divergence operator. The first equality defines $G$, the generation of wave activity $\overline{\zeta^{12}} /\left(2 \partial_{y} \bar{\zeta}_{a}\right)$. Where $G$ is positive, wave activity is generated, and the eddy flux of angular momentum associated with the rotational flow converges; where $G$ is negative, wave activity is dissipated, and the eddy flux of angular momentum associated with the rotational flow diverges. Because the bulk of the eddy flux of angular momentum is associated with the rotational (geostrophic) flow, convergence/divergence of the eddy angular momentum flux $\overline{u_{\Psi}^{\prime} v_{\Psi}^{\prime}} \cos \phi$ associated with the rotational flow is 
essentially synonymous with convergence/divergence of the overall eddy angular momentum flux $\overline{u^{\prime} v^{\prime}} \cos \phi$.

We will use

$$
\left\langle G_{e}^{+}\right\rangle=\frac{1}{\Delta \phi} \int_{-5^{\circ}}^{+5^{\circ}} \frac{1}{\Delta \sigma} \int_{\sigma_{t}+\Delta \sigma}^{\sigma_{t}} G^{+} \cos \phi d \sigma d \phi
$$

the cosine-weighted average of only the positive values $G^{+}$ of $G$ in the equatorial upper troposphere, as a measure of the wave activity generation in the equatorial region and, hence, as a measure of equatorially generated eddy angular momentum flux convergence, $-\operatorname{div}\left(\overline{u^{\prime} v^{\prime}} \cos \phi\right)_{e}$. Here, the subscript $e$ represents an average over the equatorial region between $-5^{\circ}$ and $+5^{\circ}$. Angle brackets represent an average over a fixed depth in the upper troposphere, taken from the equatorial tropopause (defined using a critical lapse rate of $2 \mathrm{~K} \mathrm{~km}^{-1}$ ) at the level $\sigma_{t}=p_{t} / p_{s}$ to the level lying $\Delta \sigma=\Delta p / p_{s}=0.3$ below it. There is one subrotating simulation $\left(\Omega=2 \Omega_{e}, \Delta_{h}=120 \mathrm{~K}, \gamma=0.7\right)$ with negative values of $\left\langle G_{e}\right\rangle$ in the averaging region such that $\left\langle G_{e}^{+}\right\rangle$is zero. We have omitted this simulation from the figures that follow, because they show the logarithm of $\left\langle G_{e}\right\rangle$, which is not a real number for this simulation.

Angular momentum divergence due to wave breaking of midlatitude baroclinic eddies can partially or fully compensate the angular momentum convergence in the equatorial region associated with equatorial wave activity generation $\left\langle G_{e}^{+}\right\rangle$. We want to quantify the angular momentum flux divergence $M=\operatorname{div}\left(\overline{u^{\prime} v^{\prime}} \cos \phi\right)_{x}$ (subscript $x$ denotes extratropical quantities) these baroclinic eddies generate when they dissipate in the tropics. We use an upper-tropospheric average of the eddy angular momentum flux divergence in the first "lobe" of divergence nearest to the equator:

$$
\left\langle M_{d}\right\rangle=\frac{1}{\Delta \phi} \int_{\phi_{2}}^{\phi_{1}} \frac{1}{\Delta \sigma} \int_{\sigma_{t}+\Delta \sigma}^{\sigma_{t}} M d \sigma d \phi .
$$

After vertically averaging over the fixed depth in the upper troposphere, we determine the latitudes bounding the first lobe of divergence for each simulation by starting at the equator and finding the latitude $\phi_{1}$ where $\langle M\rangle$ becomes positive, indicating divergence, then continuing poleward up to the latitude $\phi_{2}$ where $\langle M\rangle$ changes sign to become negative. This average over the divergence lobe closest to the equator is denoted by the subscript $d$. Divergence in this region is produced when midlatitude baroclinic eddies dissipate there, for example, through filamentation and rollup in their critical layers (Stewartson 1977; Randel and Held 1991) or through interaction with the mean-flow shear (Farrell 1987; Lindzen 1988; Huang and Robinson 1998; O'Gorman and Schneider 2007; Ait-Chaalal and

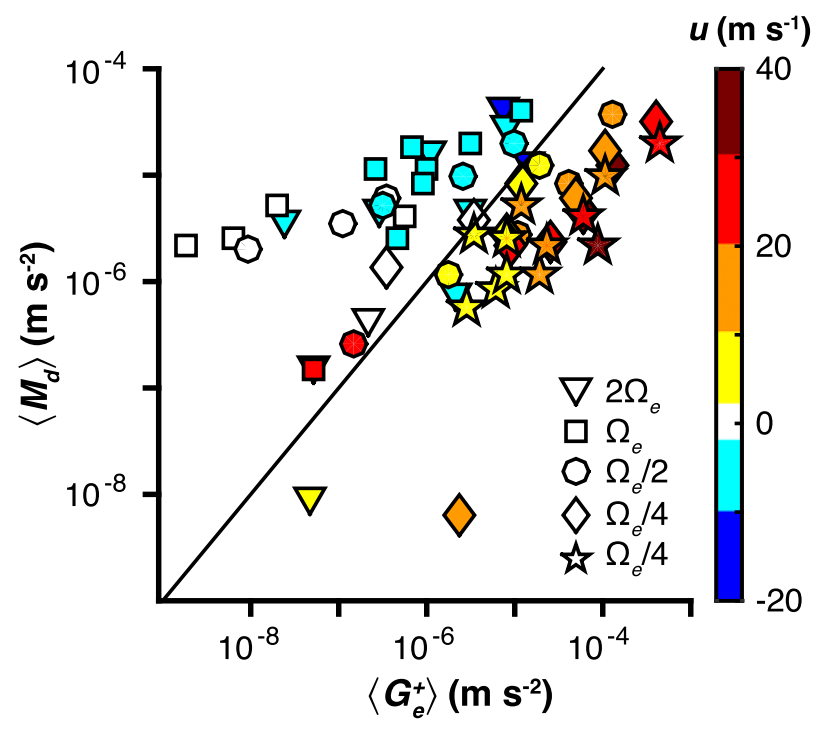

FIG. 3. All simulations shown as a function of $\left\langle G_{e}^{+}\right\rangle$and $\left\langle M_{d}\right\rangle$. In this and subsequent figures, the marker color shows the mean zonal wind averaged within $5^{\circ}$ of the equator in the upper troposphere (in the same way that $\left\langle G_{e}^{+}\right\rangle$is averaged). Positive values (red) indicate superrotation, and negative values (blue) indicate subrotation. The different symbols indicate the planetary rotation rates shown in the legend. The solid black line is the 1:1 line.

Schneider 2015). Thus, $\left\langle M_{d}\right\rangle$ provides us with a measure of wave activity dissipation associated with baroclinic waves equatorward of their midlatitude generation regions. However, the measure is imperfect in that some angular momentum flux divergence associated with midlatitude eddies in this region may be compensated by locally generated angular momentum flux convergence, or it may be augmented by divergence associated with equatorial waves.

Figure 3 displays all 59 simulations as a function of $\left\langle G_{e}^{+}\right\rangle$and $\left\langle M_{d}\right\rangle$. The black line is the one-to-one line. The different symbols indicate the planetary rotation rate for each simulation, and the colors of the symbols indicate the magnitude of the equatorial uppertropospheric zonal wind. Positive values (red) indicate superrotation, and negative values (blue) subrotation.

The simulations that lie to the right of the one-to-one line (i.e., with $\left\langle G_{e}^{+}\right\rangle>\left\langle M_{d}\right\rangle$ ) are generally superrotating, and those on the left (i.e., with $\left\langle G_{e}^{+}\right\rangle<\left\langle M_{d}\right\rangle$ ) are generally subrotating, with a couple of exceptions. That is, the ratio of the two parameters $\left\langle G_{e}^{+}\right\rangle$and $\left\langle M_{d}\right\rangle$ captures fairly well whether a given atmosphere is superrotating. It is clear that the planetary rotation rate plays a large role in controlling $\left\langle G_{e}^{+}\right\rangle$: the slower rotation rates generally lie on the right side (superrotation), and the faster ones lie on the left (subrotation).

There are two strongly superrotating simulations that lie on the left side of the line and for which the scalings 
do not work well in the following figures. These simulations both have $\Delta_{h}=30 \mathrm{~K}$ and $\gamma=0.5$. They are the kind of simulations for which we needed to increase the subgrid-scale damping to decrease the noisiness of the simulations (section 2). We found that both the calculation of $\left\langle G_{e}^{+}\right\rangle$and the equatorial wind speed in these simulations with such low baroclinicity are very sensitive to the subgrid-scale damping. For example, both simulations were more weakly superrotating in the upper troposphere with weaker damping. That subgridscale damping becomes dynamically important and leads to numerical sensitivities may explain why these simulations are inconsistent with the theory.

\section{Scaling theory}

To understand more completely under which conditions superrotation arises, we develop a scaling theory for the wave activity generation $\left\langle G_{e}^{+}\right\rangle$near the equator and for the off-equatorial eddy angular momentum flux divergence $\left\langle M_{d}\right\rangle$.

\section{a. Equatorial wave activity generation}

To determine how $\left\langle G_{e}^{+}\right\rangle$scales with mean-flow parameters, we need scalings for $R^{\prime}, \zeta^{\prime}$, and $\partial_{y} \bar{\zeta}_{a}$. Because the Rossby wave source is $R^{\prime}=R-\bar{R}$ by definition, with $R=\nabla_{h} \cdot\left(\zeta_{a} \mathbf{v}_{\chi}\right)$ according to (2), we can expand

$$
\begin{aligned}
R^{\prime} & \approx \nabla_{h} \cdot\left(\zeta^{\prime} \overline{\mathbf{v}}_{\chi}+\bar{\zeta}_{a} \mathbf{v}_{\chi}^{\prime}\right) \\
& \approx\left(\overline{\mathbf{v}}_{\chi} \cdot \nabla_{h}\right) \zeta^{\prime}+\zeta^{\prime}\left(\nabla_{h} \cdot \overline{\mathbf{v}}_{\chi}\right)+\left(\mathbf{v}_{\chi}^{\prime} \cdot \nabla_{h}\right) \bar{\zeta}_{a}+\bar{\zeta}_{a}\left(\nabla_{h} \cdot \mathbf{v}_{\chi}^{\prime}\right)
\end{aligned}
$$

where we assume that the nonlinear eddy-eddy interaction term $\zeta^{\prime} \mathbf{v}_{\chi}^{\prime}-\overline{\zeta^{\prime} \mathbf{v}_{x}^{\prime}}$ is negligible. A priori, the terms on the right-hand side of (7) are all of similar magnitude. We develop a scaling for the last term, the stretching of mean absolute vorticity by divergence fluctuations, which has traditionally been a focus of equatorial wave generation theories. It scales like the mean absolute vorticity $\bar{\zeta}_{a}$ multiplied by a scaling for the divergence of the fluctuating horizontal flow $\nabla_{h} \cdot \mathbf{v}_{\chi}^{\prime}$. For the mean absolute vorticity, we assume $\bar{\zeta}_{a} \sim \beta_{e} L_{\beta_{e}}$, and for its gradient $\partial_{y} \bar{\zeta}_{a} \sim \beta_{e}$, where $\beta_{e}=\partial f / \partial y \approx 2 \Omega / a$ is the derivative of the Coriolis parameter $f$ at the equator, and $L_{\beta_{e}}$ is a length scale of the rotational flow. The latter we will take to be the equatorial Rossby radius,

$$
L_{\beta_{e}} \sim\left(\frac{H_{e} N_{e}}{\beta_{e}}\right)^{1 / 2},
$$

where $H_{e}$ is the equatorial tropopause height and $N_{e}$ is the equatorial buoyancy frequency. For the divergence of the fluctuating horizontal flow, we can derive a scaling

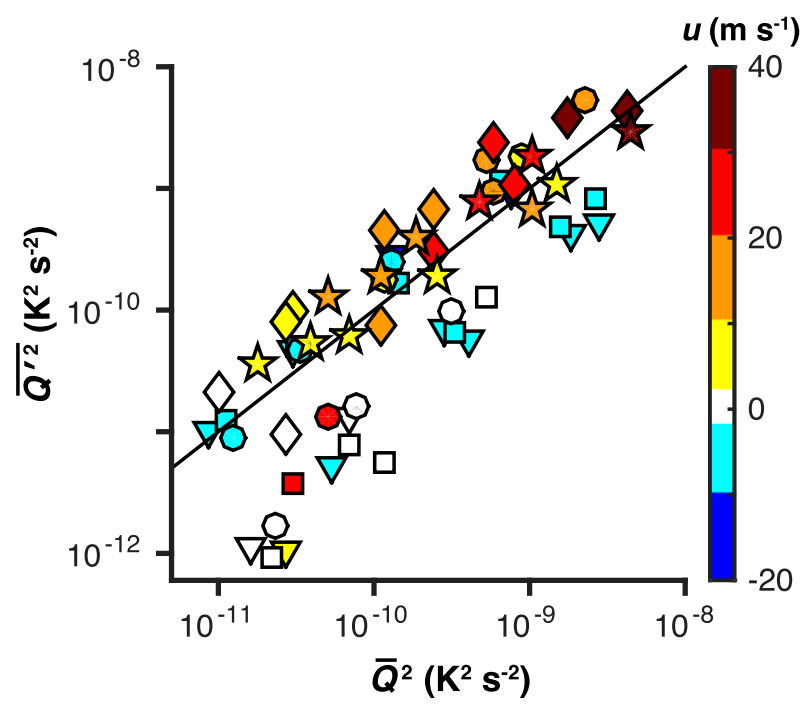

FIG. 4. Convective heating fluctuations vs mean convective heating.

from the continuity equation combined with the thermodynamic equation under the weak temperature gradient approximation (Sobel et al. 2001; Schneider and Liu 2009):

$$
\nabla_{h} \cdot \mathbf{v}_{\chi}^{\prime} \approx-\frac{\partial}{\partial z}\left(\frac{g Q^{\prime}}{\theta_{0} N_{e}^{2}}\right) \sim \frac{g Q^{\prime}}{\theta_{0} H_{e} N_{e}^{2}} .
$$

This assumes divergence fluctuations are driven primarily by diabatic (i.e., convective) heating fluctuations $Q^{\prime}$ in the upper troposphere, and the height scale over which the vertical velocity $w \sim g Q^{\prime} /\left(\theta_{0} N_{e}^{2}\right)$ diverges is proportional to the tropopause height (e.g., $\left.0.1 H_{e}\right)$. Alternative choices for this height scale are possible. For example, one might take it to be constant or use the scale height $H=R T / g$. The difference between taking the height scale to be constant and using a fraction of $H_{e}$ is quite small. However, using $H$ does not fully capture the dependence of the height scale on the stratification in our simulations.

To relate $\nabla_{h} \cdot \mathbf{v}_{\chi}^{\prime}$ to mean-flow quantities, we need to express $Q^{\prime}$ on the right-hand side of (9) in terms of a mean-flow quantity. We take $Q^{\prime}$ to scale with the temporal- and zonal-mean convective heating $\bar{Q}$, obtained from the GCM's convection scheme. This captures the heating fluctuations well, except for some simulations with very weak convective heating, where it overestimates the diabatic heating fluctuations (Fig. 4). The resulting scaling (9) of the rms divergence fluctuations in the upper troposphere, averaged in the same way and in the same region as $\left\langle G_{e}^{+}\right\rangle$and using $Q^{\prime} \sim \bar{Q}$, works well for most of the simulations, particularly the superrotating ones (Fig. 5). It overestimates the divergence for subrotating simulations with very weak equatorial divergence fluctuations. 


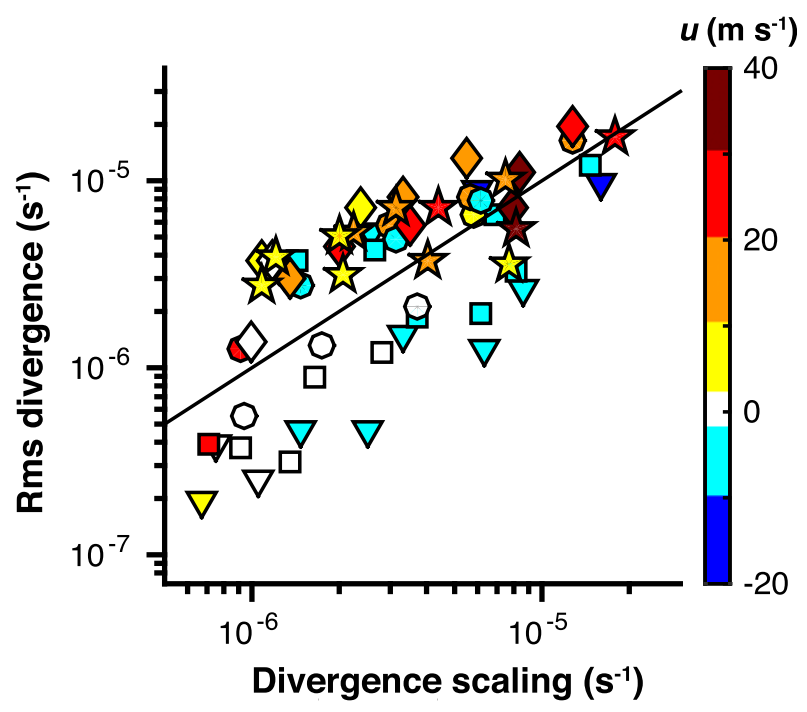

FIG. 5. Root-mean-square equatorial divergence fluctuations $\nabla_{h} \cdot \mathbf{v}_{\chi}^{\prime}$ vs the divergence scaling (9), multiplied by a constant factor 21.9 obtained from a least squares fit. The solid line is the least squares fit through the data points.

To obtain a scaling for the wave activity generation, it remains to find a scaling for the enstrophy source $\overline{R^{\prime} \zeta^{\prime}}$, given the scaling for the Rossby wave source $R^{\prime}$. We find that vorticity fluctuations $\zeta^{\prime}$ scale with the Rossby wave source $R^{\prime} \sim \bar{\zeta}_{a}\left(\nabla_{h} \cdot \mathbf{v}^{\prime}\right)$ divided by the inverse time scale given by the mean vorticity $\bar{\zeta}_{a} \sim \beta_{e} L_{\beta_{e}}$. When combining the scalings for $R^{\prime}$ and $\zeta^{\prime}$, one obtains $\zeta^{\prime} \sim \nabla_{h} \cdot \mathbf{v}^{\prime} \sim g \bar{Q} /\left(\theta_{0} H_{e} N_{e}^{2}\right)$. That is, vorticity fluctuations scale with divergence fluctuations, as might be expected in regimes where the Rossby number is $O(1)$. This implies that the other terms in (7) scale similarly to the stretching term on which we focused. The similarity of divergence and vorticity fluctuations also seems to imply that $\zeta^{\prime}$ does not depend on the planetary rotation rate; however, this is not the case. The planetary rotation rate enters the $\zeta^{\prime}$ scaling through the heating rate $Q^{\prime}$, which decreases with increasing rotation rate, empirically as $Q^{\prime} \sim \Omega^{-3 / 4}$ between $\Omega=\Omega_{e}$ and $\Omega_{e} / 4$ (Fig. 6) in our simulations. For the highest and lowest rotation rates, the dependence of $Q^{\prime}$ on rotation rate becomes weaker. In general, Fig. 6 shows that simulations with lower planetary rotation rates have stronger mean equatorial heating rates and convective heating rate fluctuations, as is to be expected given that lower planetary rotation rates necessitate weaker temperature gradients and hence stronger mean meridional circulations to transport heat near the equator (Held and Hou 1980). Correspondingly, divergence and vorticity fluctuations generally strengthen as the rotation rate decreases.

Putting this all together, we have a scaling for the equatorial wave activity source:

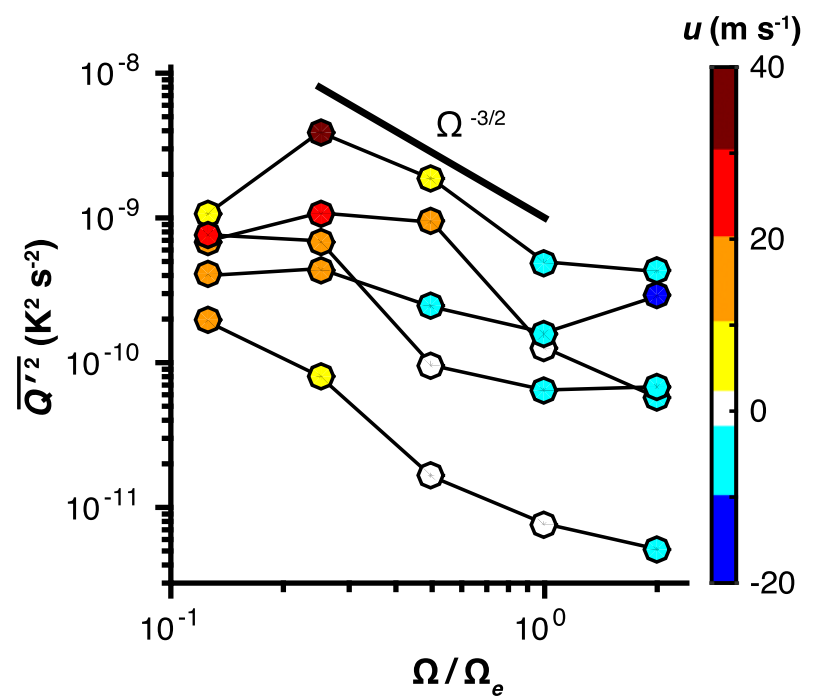

FIG. 6. Variance of convective heating fluctuations $Q^{\prime}$ vs planetary rotation rate $\Omega$ for five series of simulations holding $\Delta_{h}$ and $\gamma$ constant and varying the rotation rate (each series is connected by a black line).

$$
\left\langle G_{e}^{+}\right\rangle \sim L_{\beta_{e}}\left(\nabla_{h} \cdot \mathbf{v}^{\prime}\right)^{2} \sim \frac{g^{2} \bar{Q}^{2}}{\theta_{0}^{2} H_{e}^{3 / 2} N_{e}^{7 / 2} \beta_{e}^{1 / 2}}
$$

Figure 7 shows $\left\langle G_{e}^{+}\right\rangle$versus the scaling (10), with the right-hand side multiplied by a factor of 89.3 , obtained from a least squares fit. This factor is quite large because we have suppressed an explicit small factor multiplying the tropopause height $H_{e}$ in the height scale (e.g., $0.1 H_{e}$ ) of vertical velocity divergence in (9), which would enter the scaling (10) as an inverse square (e.g., as $0.1^{-2}$ ). Generally, the scaling captures the variations of the wave activity source over approximately three orders of magnitude quite well. The most significant departures from a one-to-one relationship occur for subrotating simulations with weak pole-to-equator temperature gradients, for which the scaling overestimates $\left\langle G_{e}^{+}\right\rangle$in similar ways as the divergence scaling (9) overestimates actual divergence fluctuations (cf. Fig. 5).

\section{b. Baroclinic angular momentum flux divergence}

The baroclinic angular momentum fluxes originating in the extratropics strengthen with increasing baroclinicity, for example, increasing pole-to-equator temperature contrasts or decreasing static stability. Schneider and Walker (2008) showed that, in simulations of dry atmospheres similar to ours, the eddy angular momentum flux divergence scales with the mean available potential energy (MAPE) per unit mass in the troposphere and inversely with the width $L_{\mathrm{bc}}$ of the baroclinic zone over which MAPE is calculated: 


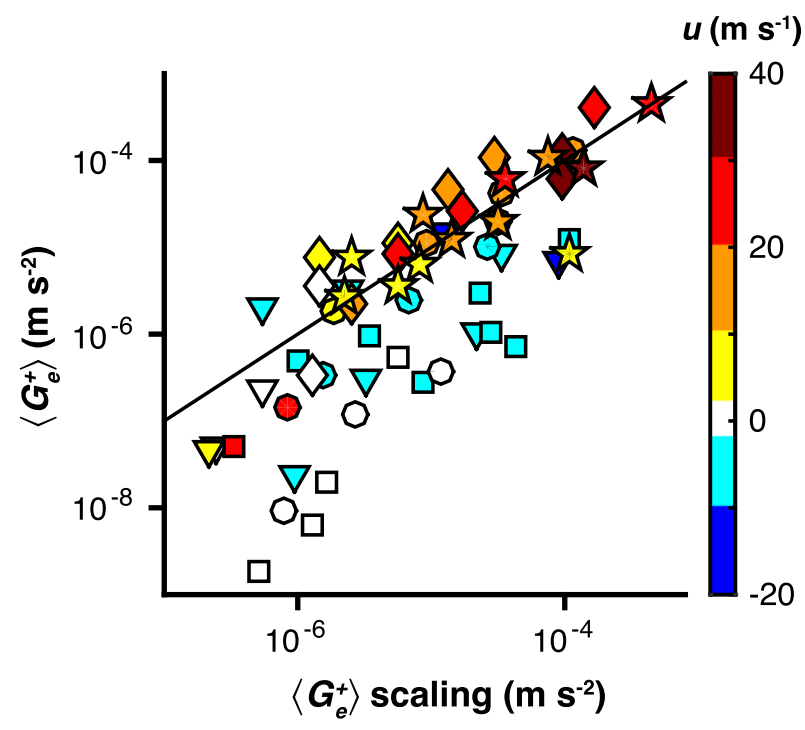

FIG. 7. Equatorial wave activity source $\left\langle G_{e}^{+}\right\rangle$vs the scaling (10), multiplied by a constant factor 89.3 obtained from a least squares fit. The solid line is the least squares fit through the data points.

$$
\left\langle M_{d}\right\rangle \sim \frac{\text { MAPE }}{L_{\mathrm{bc}}} .
$$

Here, MAPE per unit mass is approximately

$$
\mathrm{MAPE} \approx \frac{g^{2}}{24 N_{x}^{2}}\left(\frac{\Delta \theta}{\theta_{0}}\right)^{2}
$$

where $\Delta \theta$ is the meridional potential temperature contrast across a baroclinic zone, $\theta_{0}$ is a reference value of near-surface potential temperature, and $N_{x}$ is the extratropical buoyancy frequency (Schneider 1981; Schneider and Walker 2008). For simplicity, we have omitted the dependence of MAPE on the supercriticality $S_{c}$, which was discussed by Schneider and Walker (2008). Including it changes the scaling only marginally for the simulations we consider here. The width of the baroclinic zone only varies by a factor of 2 in our simulations, because the width of this zone is limited by the size of the planet for simulations with low planetary rotation rate like the ones studied here. Hence, here we use a constant length scale $L_{\mathrm{bc}}=\pi a / 4$, but for faster rotation rates it would need to be reduced, for example, scaling like the Rossby radius, $L_{\mathrm{bc}} \sim L_{R} \sim \Omega^{-1}$. Combining these estimates and omitting nondimensional factors gives

$$
\left\langle M_{d}\right\rangle \sim \frac{g^{2}}{L_{\mathrm{bc}} N_{x}^{2}}\left(\frac{\Delta \theta}{\theta_{0}}\right)^{2}
$$

This scaling captures the dependence of the baroclinic eddy angular momentum flux divergence in most

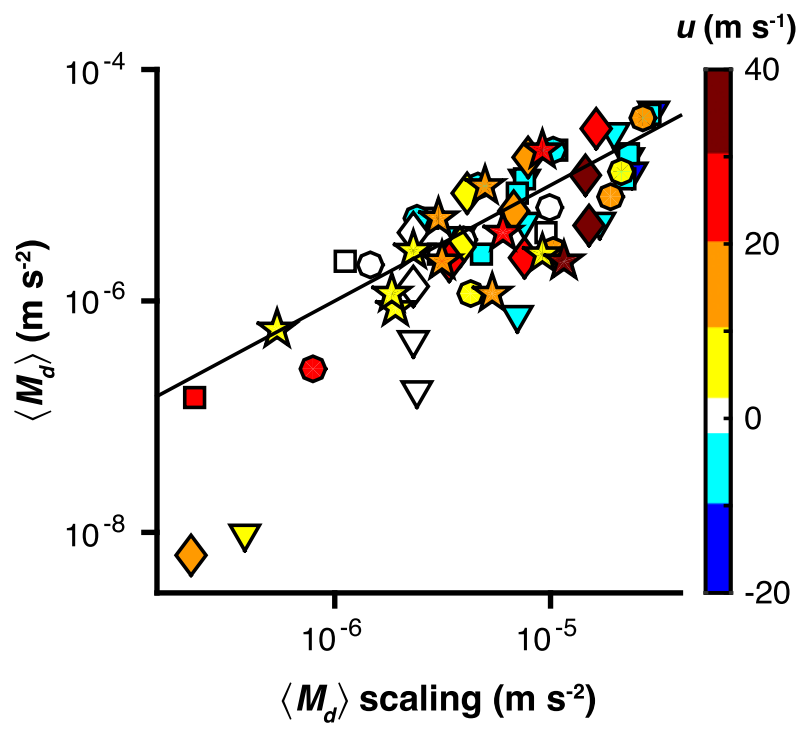

FIG. 8. Eddy angular momentum flux divergence $\left\langle M_{d}\right\rangle$ vs the scaling (13), multiplied by a constant factor 0.174 obtained from a least squares fit. The solid line is the least squares fit through the data points.

simulations reasonably well, except in some very weakly baroclinic simulations, in which it overestimates $\left\langle M_{d}\right\rangle$ (Fig. 8). A least squares fit suggests that the right-hand side needs to be multiplied by 0.174 to give a good fit to the simulation results.

Combing the estimate for the equatorial wave activity generation (angular momentum flux convergence) with that for the baroclinic angular momentum flux divergence, we can introduce a nondimensional number

$$
S_{r}=\frac{\left\langle G_{e}^{+}\right\rangle}{\left\langle M_{d}\right\rangle} \sim \alpha \frac{L_{\mathrm{bc}} N_{x}^{2}}{\left(H_{e}^{3} N_{e}^{3} \beta_{e}\right)^{1 / 2}}\left(\frac{\bar{Q}}{N_{e} \Delta \theta}\right)^{2} .
$$

The factor $\alpha \approx 500$ is the ratio of the nondimensional factors in the scalings for $\left\langle G_{e}^{+}\right\rangle$and $\left\langle M_{d}\right\rangle$. (Again, this factor $\alpha$ is large primarily because we suppressed an explicit small factor multiplying the tropopause height $H_{e}$ in the height scale of vertical velocity divergence.) The nondimensional quantity $S_{r}$ quantifies an atmosphere's propensity for superrotation in terms of meanflow quantities. When $S_{r} \gtrsim 1$, an atmosphere will usually superrotate, and, when $S_{r} \lesssim 1$, it will usually subrotate. This tells us that atmospheric superrotation is favored for stronger convective heating at the equator $\bar{Q}$, reduced meridional temperature gradients $\Delta \theta$, lower planetary rotation rates $\left(\beta_{e} \sim \Omega\right)$, and weaker equatorial $\left(N_{e}\right)$ or stronger extratropical $\left(N_{x}\right)$ static stability. Because the convective heating generally strengthens with decreasing rotation rate, the dependence of $S_{r}$ on $\Omega$ is stronger than that occurring explicitly through the $\beta_{e}^{-1 / 2}$ term. 

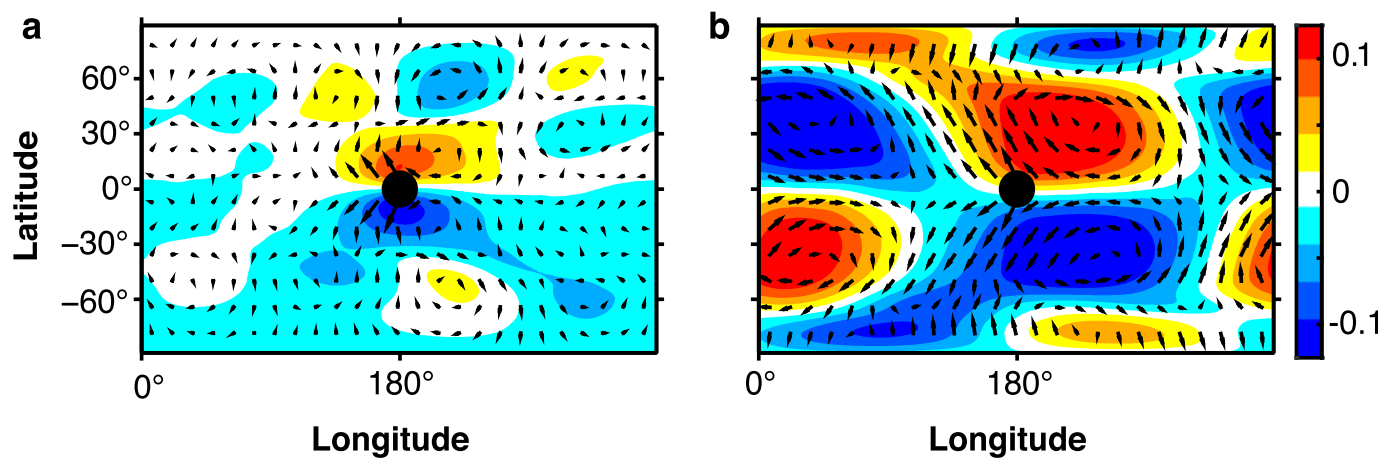

FIG. 9. Correlation coefficients between divergence fluctuations at a reference point on the equator (black dot) and horizontal streamfunction fluctuations (colors) and wind (arrows) at $300 \mathrm{hPa}$. (a) Superrotating simulation with $\Omega=\Omega_{e} / 2, \Delta_{h}=240 \mathrm{~K}$, and $\gamma=0.9$. (b) Superrotating simulation with $\Omega=\Omega_{e} / 8, \Delta_{h}=120 \mathrm{~K}$, and $\gamma=0.7$ (see second row of Fig. 1). Arrows represent correlation coefficients between wind fluctuations and divergence fluctuations at the equator. An arrow pointing right corresponds to a positive correlation for $u^{\prime}$ and zero correlation for $v^{\prime}$; an arrow pointing upward corresponds to a positive correlation for $v^{\prime}$ and zero correlation for $u^{\prime}$. The lengths of the arrows indicate the magnitude of the correlations.

\section{Discussion}

\section{a. Mechanisms and origin of parameter dependences}

Although idealized, the simulations performed here have the basic ingredients to produce superrotation in terrestrial atmospheres. The most important quantities controlling whether superrotation occurs or not in our simulations are the planetary rotation rate and the meridional temperature gradient, with, for example, the static stability in the tropics and extratropics playing secondary roles.

The dependence of $S_{r}$ on rotation rate comes primarily from the equatorial wave activity generation $\left\langle G_{e}^{+}\right\rangle$. The extratropical $\left\langle M_{d}\right\rangle$ will depend on rotation rate for faster rotation rates $\left(\left\langle M_{d}\right\rangle \sim \Omega\right)$ because the width of the baroclinic zone $L_{\mathrm{bc}}$ depends on rotation rate, like the Rossby radius $L_{R} \sim \Omega^{-1}$. For the simulations discussed here, however, the planetary scale limits this rotation rate dependence. The equatorial $\left\langle G_{e}^{+}\right\rangle \sim L_{\beta_{e}}\left(\nabla_{h} \cdot \mathbf{v}^{\prime}\right)^{2}$ decreases with rotation rate for two primary reasons: The mean-square divergence $\left(\nabla_{h} \cdot \mathbf{v}_{\chi}^{\prime}\right)^{2} \sim \bar{Q}^{2}$ depends on rotation rate because the squared diabatic heating rate does, roughly like $\sim \Omega^{-3 / 2}$ in our simulations. Additionally, the equatorial Rossby radius depends on rotation rate, $L_{\beta_{e}} \sim \Omega^{-1 / 2}$.

Thus, for faster rotation rates, the baroclinic eddy angular momentum flux divergence increases with rotation rate primarily because eddies get smaller and the angular momentum fluxes become more concentrated in narrower baroclinic zones. Increases in meridional temperature contrasts because of reduced efficiency of poleward energy transport also modify the baroclinic eddy angular momentum flux divergence (Schneider and Walker 2008). By contrast, the equatorial wave activity generation decreases with rotation rate both because the equatorial Rossby radius decreases and the convective heating fluctuations and divergence fluctuations weaken. For our simulations, the equatorial wave activity generation effect dominates and leads to $S_{r} \sim \Omega^{-2}$ : that is, a strongly increasing propensity for superrotation with decreasing rotation rate. (For faster rotation rates, this amplifies to $S_{r} \sim \Omega^{-3}$ when, additionally, the shrinking width of the baroclinic zone is taken into account.) This may account for the fact that slowly rotating terrestrial atmospheres (e.g., Venus's) tend to exhibit superrotation.

The dependence of $S_{r}$ on the meridional temperature contrast $\Delta \theta$ enters explicitly only through the scaling for $\left\langle M_{d}\right\rangle$, which depends on MAPE $\sim(\Delta \theta)^{2}$, yielding $S_{r} \sim(\Delta \theta)^{-2}$. Thus, when meridional temperature gradients are weak, there is less angular momentum flux divergence near the equator by midlatitude baroclinic eddies, and the propensity for superrotation increases. It is worthwhile noting that, when $\Delta \theta$ is decreased in our simulations, the polar temperature remains constant, and the equatorial and globally averaged temperatures decrease, decreasing the equatorial tropopause height $H_{e}$ (Thuburn and Craig 2000; Schneider 2004) and adding to the propensity for superrotation. The meridional temperature contrast and the rotation rate may also both affect the static stability in the extratropics and near the equator, which, in themselves, have opposing effects on $S_{r} \sim N_{x}^{2} N_{e}^{-7 / 2}$.

To look at the equatorial wave structures responsible for the generation of superrotation more closely, Fig. 9 shows the correlation coefficient between equatorial divergence fluctuations at a reference point and horizontal streamfunction and wind fluctuations at $300 \mathrm{hPa}$ 
for two superrotating simulations, one with half Earth's rotation rate $\left(\Omega=\Omega_{e} / 2, \Delta_{h}=240 \mathrm{~K}\right.$, and $\left.\gamma=0.9\right)$, and one with even slower planetary rotation $\left(\Omega=\Omega_{e} / 8\right.$, $\Delta_{h}=120 \mathrm{~K}$, and $\gamma=0.7$; see second row of Fig. 1$)$. The reference point is indicated by a black dot at the equator at $180^{\circ}$ longitude. The correlation coefficients between divergence fluctuations at the equator and, on the one hand, horizontal streamfunction fluctuations (colors) and, on the other hand, wind fluctuations (arrows), indicate a Rossby (rotational) wave structure for both cases (Matsuno 1966). The Rossby wave has zonal velocities symmetric about the equator and meridional velocities antisymmetric about the equator. The equatorial Rossby radius, which determines the decay of meridional velocity correlations away from the equator, appears to extend over much of a hemisphere for the slowly rotating simulation $\left(\Omega=\Omega_{e} / 8\right)$, but it is more confined to the equator in the more slowly rotating simulation $\left(\Omega=\Omega_{e} / 2\right)$. Similar equatorial wave features were seen in the giant-planet simulations of Schneider and Liu (2009) and Liu and Schneider (2010, 2011).

The correlations between wind fluctuations and divergence fluctuations at the equator (tilt of arrows) indicate angular momentum transport toward the equator. They also show that no more than the usual tilt of phase lines with latitude is needed to generate the angular momentum transport. It is not necessary to see outright meridional propagation of wave packets for angular momentum transport to occur. It suffices to have a meridional group velocity away from the equator, as indicated by the tilt of phase lines, accompanied by dissipation of waves preferentially in the off-equatorial wings: for example, by critical-layer rollup or shearing by the mean flow (e.g., Farrell 1987; Lindzen 1988; Huang and Robinson 1998; O'Gorman and Schneider 2007; Ait-Chaalal and Schneider 2015). This overall picture is consistent with our theoretical considerations, which assign primary importance to equatorial Rossby waves and their equatorward angular momentum transport in the generation of superrotation.

Figure 10 shows the eddy angular momentum flux cospectra (black contours for positive and blue contours for negative fluxes) versus latitude at the level $\sigma=0.3$ for a subrotating and a superrotating simulation (corresponding to the first and second rows of Fig. 1). The thick black line is the mean zonal wind at the same level. The subrotating simulation shows similar features to what we see on Earth (Randel and Held 1991): baroclinic eddies generated in midlatitudes have phase speeds of order $10 \mathrm{~m} \mathrm{~s}^{-1}$ and dissipate near their critical latitudes, which produces convergence of eddy angular momentum fluxes in midlatitudes and divergence primarily on the equatorward flanks of the jets. For the superrotating simulation, we see something different: Eddies have phase speeds of order $1 \mathrm{~m} \mathrm{~s}^{-1}$ (i.e., they are more strongly westward relative to the mean flow); their associated angular momentum fluxes converge near the equator. The eddies do not seem to dissipate near their critical latitudes but instead produce angular momentum flux convergence near the equator through interaction with the mean-flow shear, consistent with the tilted phase lines seen in Fig. 9 (e.g., Farrell 1987; Lindzen 1988; Huang and Robinson 1998; O'Gorman and Schneider 2007; Ait-Chaalal and Schneider 2015).

\section{b. Relation to prior work}

Our scaling theory and simulation results are consistent with previous simulations of superrotating terrestrial atmospheres. The scaling theory provides a unifying framework within which the generation of superrotation, for example, in slowly rotating (e.g., Del Genio et al. 1993; Del Genio and Zhou 1996; Walker and Schneider 2006) or strongly convective (e.g., Schneider and Liu 2009; Caballero and Huber 2010; Liu and Schneider 2011) atmospheres can be interpreted as arising from a common set of principles. It provides a quantitative criterion to determine when superrotation occurs, which is more generally applicable than previous criteria.

For example, Mitchell and Vallis (2010) and Potter et al. (2014) argued that terrestrial atmospheres transition from subrotation to superrotation when the thermal Rossby number

$$
\mathrm{Ro}_{T}=\frac{R_{a} \Delta_{h}}{(2 \Omega a)^{2}}
$$

exceeds 1 (gas constant of air $R_{a}$ and pole-equator temperature contrast in radiative equilibrium $\Delta_{h}$ ). The mechanism for generation and maintenance of superrotation at large thermal Rossby numbers is posited to be the development of a global-scale disturbance, involving Kelvin waves (Mitchell and Vallis 2010; Pinto and Mitchell 2014; Potter et al. 2014). By contrast, equatorial Rossby waves are involved in the generation and maintenance of superrotation in our theory and simulations, and they have substantial amplitude primarily within an equatorial Rossby radius, as seen in Fig. 9. Among other differences between our general circulation models used to test the theories, the main distinction is that we use one in which the radiative equilibrium is statically unstable and that employs a convection scheme to provide vertical heat transport. Mitchell and Vallis (2010) and Potter et al. (2014) use a model with a statically stable radiative equilibrium. However, given that our arguments merely require the 

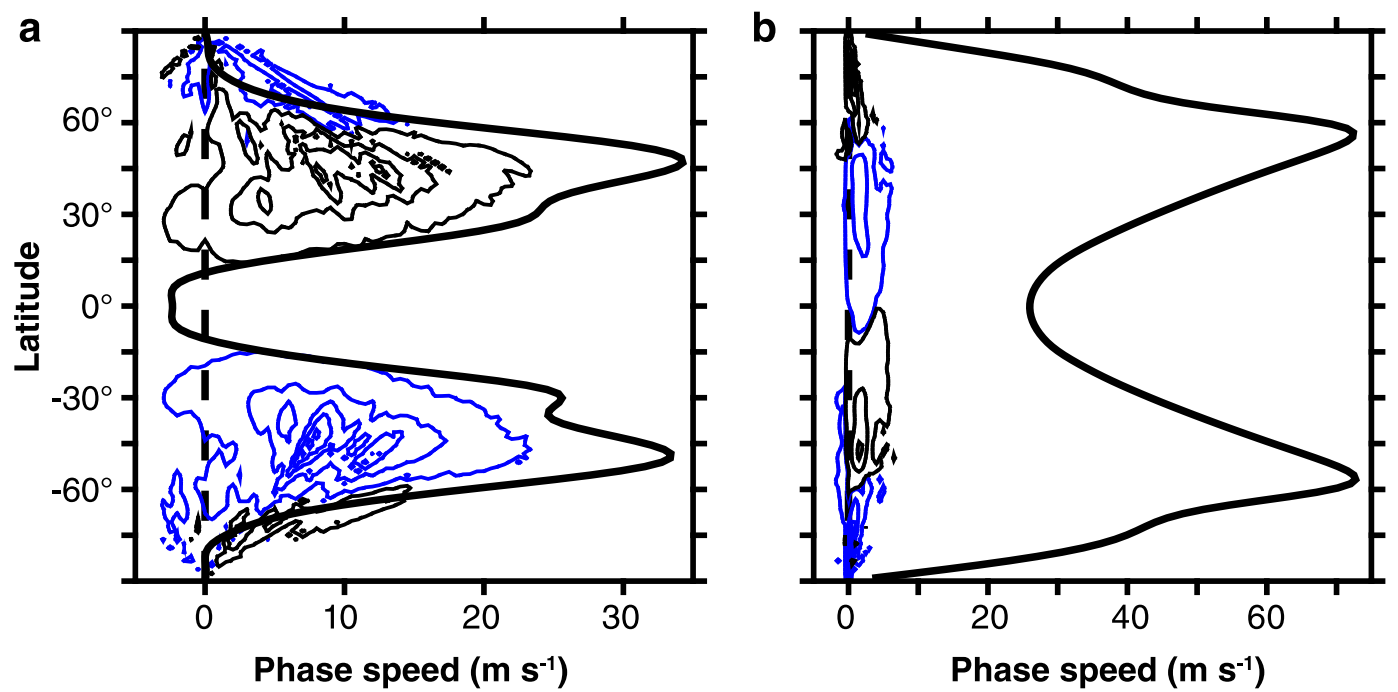

FIG. 10. Eddy angular momentum flux cospectra vs latitude at $\sigma=0.3$. (a) A subrotating simulation $\left(\Omega=\Omega_{e}\right.$, $\Delta_{h}=120 \mathrm{~K}$, and $\gamma=0.7$; see first row of Fig. 1). (b) The same superrotating simulation as in Fig. $9 \mathrm{~b}\left(\Omega=\Omega_{e} / 8\right.$, $\Delta_{h}=120 \mathrm{~K}$, and $\gamma=0.7$; see second row of Fig. 1). Black and blue contours correspond to positive and negative angular momentum fluxes, respectively, and the thick black line shows the mean zonal wind at the same level.

presence of divergence fluctuations to generate Rossby waves, irrespective of how the divergence fluctuations are created, this distinction between the models may not be essential: even the model with a statically stable radiative equilibrium has preferential tropospheric heating and a divergent circulation in low latitudes. The divergent circulation likely fluctuates, with divergence fluctuations that may scale with the mean diabatic heating rate and that can generate Rossby waves in essentially the same way we described.

To compare how well $\mathrm{Ro}_{T}$ captures when superrotation occurs, Fig. 11a shows the equatorial wind speed in the upper troposphere versus $\mathrm{Ro}_{T}$ for all of our simulations. The symbols indicate the planetary rotation rate (as in Fig. 3), and the colors indicate the value of $\Delta_{h}$, with blue representing low values and red representing high values. Simulations with high $\mathrm{Ro}_{T}$ do tend to be superrotating, but there is no clear threshold for superrotation. The same holds when we use the same diagnostic for superrotation (vertical mean of zonal wind) as in Mitchell and Vallis (2010) and Potter et al. (2014). Additionally, there are numerous simulations that have low $\mathrm{Ro}_{T}$ yet are superrotating.

Simulations in three series are connected with thick green lines in Fig. 11a. These simulations have the same values of $\gamma$ and $\Omega$, and the dots along each green line show what happens as $\Delta_{h}$ alone is varied. For the simulations that are subrotating, decreasing the poleequator temperature contrast (and so decreasing $\mathrm{Ro}_{T}$ by definition) can lead to superrotation, as shown by two of the connected series of simulations in Fig. 11a and as seen in Fig. 1 (cf. rows 3 and 4). This behavior is in line with the theory presented in this paper, that decreasing baroclinicity in midlatitudes while holding other parameters constant will increase $S_{r}$ and eventually lead to superrotation. However, it runs counter to the notion that the thermal Rossby number alone would generally predict when superrotation occurs. For strongly superrotating simulations, decreasing $\Delta_{h}$ has the opposite effect: it weakens the superrotation, as shown by the connected simulations near the top right of Fig. 11. This is more in line with the notion that the thermal Rossby number predicts when superrotation occurs.

The nondimensional number $S_{r}$ developed here diagnoses more generally whether an atmosphere will be super- or subrotating. It shows a clearer threshold for superrotation at $S_{r}=1$, with only the two very weakly baroclinic simulations discussed in section $3 b$ deviating from the expected behavior (Fig. 11b). However, $S_{r}$ does not provide any information on how strong or weak any superrotation will be. For example, as mentioned previously and as shown in Fig. 11, increasing $S_{r}$ beyond $S_{r} \sim 1$ by decreasing $\Delta_{h}$ actually weakens the superrotation. Scaling arguments relating the strength of superrotating jets to their widths and to the static stability in the equatorial region were provided and tested in Liu and Schneider (2010) and Liu and Schneider (2011).

\section{c. Limitations and possible extensions}

Our simulations and scaling theory ignored several factors that are known to affect whether superrotation occurs. For example, we ignored the seasonal cycle. Yet 

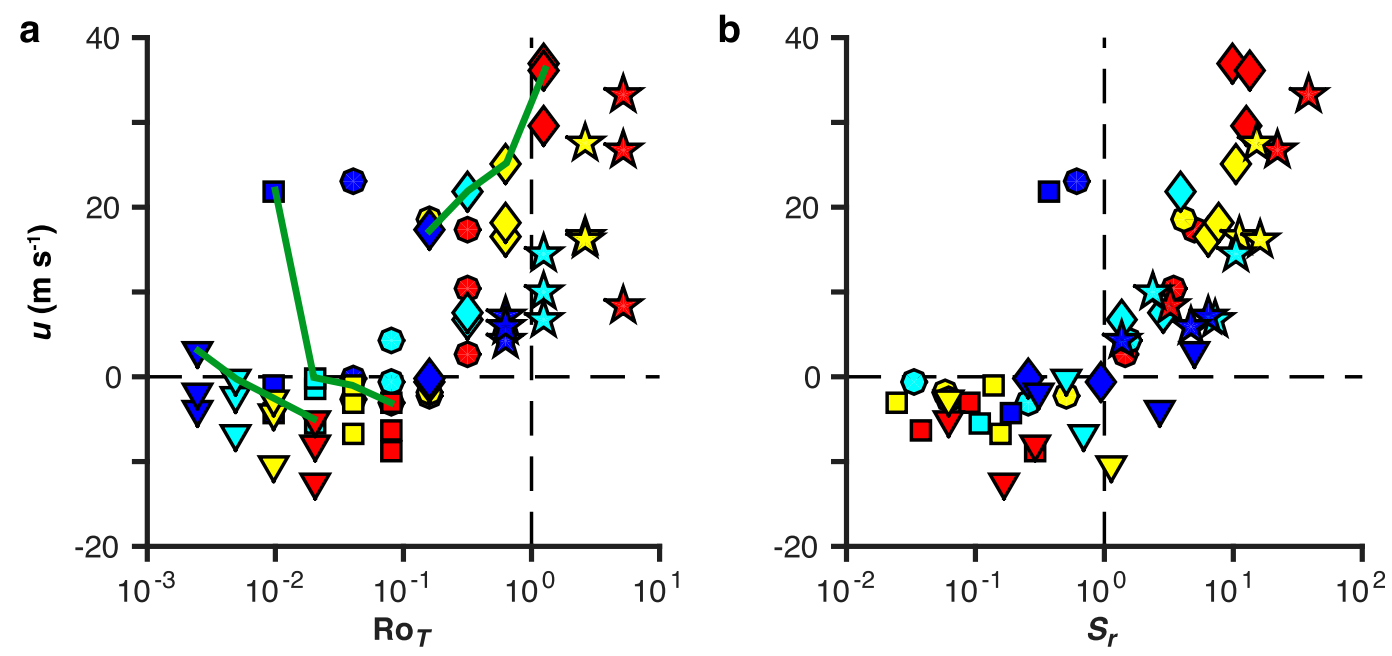

FIG. 11. Equatorial zonal wind in the upper troposphere vs (a) $\operatorname{Ro}_{T}=R_{a} \Delta_{h} /(2 a \Omega)^{2}$ and (b) $S_{r}=\left\langle G_{e}^{+}\right\rangle /\left\langle M_{d}\right\rangle$. Symbols are as in Fig. 3. Colors represent the value of $\Delta_{h}$ (blue is $30 \mathrm{~K}$, cyan is $60 \mathrm{~K}$, yellow is $120 \mathrm{~K}$, and red is $240 \mathrm{~K})$. Simulations that lie above the horizontal dashed line $\left(u=0 \mathrm{~m} \mathrm{~s}^{-1}\right)$ are superrotating. The vertical dashed lines indicate where $\operatorname{Ro}_{T}=1$ or $S_{r}=1$.

the seasonal cycle decelerates the zonal wind in the equatorial upper troposphere in the annual mean, because a Hadley circulation whose ascending branch is displaced off the equator is associated with equatorial easterlies (e.g., Lindzen and Hou 1988; Lee 1999; Kraucunas and Hartmann 2005; Mitchell et al. 2014). This disfavors superrotation for planets with nonzero obliquities. Additionally, the convective wave activity generation $G$ in the presence of a seasonal cycle would be maximal off the equator for part of the year, decreasing the numerator of $S_{r}$ and likewise disfavoring superrotation. A more general scaling theory should take into account the seasonal cycle, as well as the vertical advection of angular momentum, which becomes important seasonally (Shell and Held 2004; Kraucunas and Hartmann 2005).

We also focused on transient sources of equatorial Rossby waves, rather than stationary sources, which in several previous studies have been shown to be able to generate superrotation if they are strong enough (e.g., Suarez and Duffy 1992; Saravanan 1993; Joshi et al. 1997; Kraucunas and Hartmann 2005; Merlis and Schneider 2010; Arnold et al. 2012; Pierrehumbert 2011). Our scaling theory can be extended to take stationary equatorial wave activity generation into account by considering how the stationary divergence perturbation and equatorial wave activity generation scale with parameters controlling the strength of the stationary wave source (e.g., Merlis and Schneider 2011). In particular, it is to be expected that the importance of equatorial wave activity generation owing to stationary heat sources increases in importance relative to baroclinic eddy angular momentum flux divergence as the planetary rotation rate decreases for the same reasons the importance of transient equatorial wave activity generation increases. Thus, we expect that stationary heat sources (e.g., stellar heating focused on a substellar point on tidally locked planets) more easily lead to equatorial superrotation on slowly rotating planets.

\section{Conclusions}

We have presented simulations and a scaling theory that establish conditions under which superrotation occurs in terrestrial atmospheres. By varying the planetary rotation rate, the pole-equator temperature contrast in radiative equilibrium, and a scaling parameter for the convective lapse rate, we generated a wide range of atmospheric flows, some superrotating and some subrotating.

The theory presented here is based on a simple idea, going back to Saravanan (1993), about two competing sources for eddy angular momentum flux convergence at the equator. The first is a source at the equator: Rossby waves generated by convective heating fluctuations. As these waves dissipate preferentially away from the equator-whether after propagation of wave packets or by preferential dissipation in the off-equatorial wings of the waves (e.g., by shearing through the mean flow) they converge angular momentum into the equatorial region, increasing the propensity for superrotation. The other source is baroclinic instability in midlatitudes, which generates Rossby waves in midlatitudes that dissipate farther equatorward, thus extracting momentum from lower latitudes. This mechanism decreases the 
propensity for superrotation. Quantifying the magnitude of the two mechanisms, introducing their nondimensional ratio $S_{r}$, and developing a scaling for it in terms of mean-flow quantities leads to our theory and sheds light on the conditions under which superrotation occurs. Our simulation results and scaling theory show that:

1) Superrotation occurs when the eddy angular momentum flux convergence associated with equatorial wave activity generation exceeds eddy angular momentum flux divergence near the equator produced by midlatitude baroclinic eddies (i.e., $S_{r} \geqslant 1$ ).

2) Superrotation is favored for low planetary rotation rates and/or strong diabatic heating.

3) Superrotation is favored when midlatitude baroclinicity is weak.

Our simulations confirm that superrotation is preferred for slowly rotating planets like Venus and Titan, a result that was already obtained by Del Genio et al. (1993) and Del Genio and Zhou (1996) in a similar set of simulations. The scaling arguments presented here help us understand why slowly rotating planets exhibit superrotation. In our simulations, equatorial convective heating fluctuations strengthen with decreasing rotation rate, generating waves that transport momentum upgradient toward the equator, leading to superrotation. Such convective heating fluctuations may play a role on Venus, in the shallow convective layers observed in the upper troposphere (Markiewicz et al. 2007). The equatorial wave activity generation strengthens as the planetary rotation rate decreases primarily because the diabatic heating rate strengthens. Thus, similar arguments may also apply for other mechanisms that increase the diabatic heating or more generally, divergence fluctuations at the equator. For example, stationary or nearly stationary heat sources that generate equatorial Rossby waves have been suggested to play a role on Venus near the subsolar point (Gierasch et al. 1997).

Strengthening equatorial convective heating fluctuations may also explain why some Earth climate models exhibit a transition to superrotation under extreme global warming (e.g., Caballero and Huber 2010). In this scenario, convective heating at the equator strengthens because of greenhouse gas forcing, and meridional temperature gradients decrease because of polar amplification of the warming. Both factors favor superrotation.

Acknowledgments. Some of the scaling results in this paper were presented at the 18th Conference on Atmospheric and Oceanic Fluid Dynamics in 2011. The research was supported by the U.S. National Science
Foundation through Grant AGS-1049201 and a Graduate Research Fellowship.

\section{REFERENCES}

Ait-Chaalal, F., and T. Schneider, 2015: Why eddy momentum fluxes are concentrated in the upper troposphere. J. Atmos. Sci., 72, 1585-1604, doi:10.1175/JAS-D-14-0243.1.

Andrews, D. G., and M. E. Mcintyre, 1976: Planetary waves in horizontal and vertical shear: The generalized Eliassen-Palm relation and the mean zonal acceleration. J. Atmos. Sci., 33, 2031-2048, doi:10.1175/1520-0469(1976)033<2031:PWIHAV>2.0.CO;2.

_ , and _ 1978: An exact theory of nonlinear waves on a Lagrangian-mean flow. J. Fluid Mech., 89, 609-646, doi:10.1017/S0022112078002773.

Arnold, N. P., E. Tziperman, and B. Farrell, 2012: Abrupt transition to strong superrotation driven by equatorial wave resonance in an idealized GCM. J. Atmos. Sci., 69, 626-640, doi:10.1175/JAS-D-11-0136.1.

Caballero, R., and M. Huber, 2010: Spontaneous transition to superrotation in warm climates simulated by CAM3. Geophys. Res. Lett., 37, L11701, doi:10.1029/2010GL043468.

Charney, J. G., 1963: A note on large-scale motions in the tropics. J. Atmos. Sci. 20, 607-609, doi:10.1175/1520-0469(1963)020<0607: ANOLSM $>2.0 . \mathrm{CO} ; 2$.

Del Genio, A. D., and W. Zhou, 1996: Simulations of superrotation on slowly rotating planets: Sensitivity to rotation and initial condition. Icarus, 120, 332-343, doi:10.1006/icar.1996.0054.

,-- , and T. P. Eichler, 1993: Equatorial superrotation in a slowly rotating GCM: Implications for Titan and Venus. Icarus, 101, 1-17, doi:10.1006/icar.1993.1001.

Edmon, H. J., Jr., B. J. Hoskins, and M. E. McIntyre, 1980: Eliassen-Palm cross sections for the troposphere. J. Atmos. Sci., 37, 2600-2616, doi:10.1175/1520-0469(1980)037<2600: EPCSFT>2.0.CO;2.

Farrell, B., 1987: Developing disturbances in shear. J. Atmos. Sci., 44, 2191-2199, doi:10.1175/1520-0469(1987)044<2191: DDIS $>2.0 . \mathrm{CO} ; 2$.

Garner, S. T., D. M. W. Frierson, I. M. Held, O. Pauluis, and G. K. Vallis, 2007: Resolving convection in a global hypohydrostatic model. J. Atmos. Sci., 64, 2061-2075, doi:10.1175/JAS3929.1.

Gierasch, P., and Coauthors, 1997: The general circulation of the Venus atmosphere: An assessment. Venus II: Geology, Geophysics, Atmosphere, and Solar Wind Environment, S. W. Bougher, D. M. Hunten, and R. J. Phillips, Eds., The University of Arizona Press, 459-500.

Held, I. M., 1975: Momentum transport by quasi-geostrophic eddies. J. Atmos. Sci., 32, 1494-1497, doi:10.1175/ 1520-0469(1975)032<1494:MTBQGE > 2.0.CO;2.

_ 1999: Equatorial superrotation in Earth-like atmospheric models. [Available online at http://www.gfdl.noaa.gov/ cms-filesystem-action/user_files/ih/lectures/super.pdf.]

— in a nearly inviscid atmosphere. J. Atmos. Sci., 37, 515-533, doi:10.1175/1520-0469(1980)037<0515:NASCIA > 2.0.CO;2.

Hide, R., 1969: Dynamics of the atmospheres of the major planets with an appendix on the viscous boundary layer at the rigid bounding surface of an electrically conducting rotating fluid in the presence of a magnetic field. J. Atmos. Sci., 26, 841-853, doi:10.1175/1520-0469(1969)026<0841:DOTAOT>2.0.CO;2.

Huang, H.-P., and W. A. Robinson, 1998: Two-dimensional turbulence and persistent zonal jets in a global barotropic model. 
J. Atmos. Sci., 55, 611-632, doi:10.1175/1520-0469(1998)055<0611 TDTAPZ $>2.0 . \mathrm{CO} ; 2$.

Joshi, M., R. Haberle, and R. Reynolds, 1997: Simulations of the atmospheres of synchronously rotating terrestrial planets orbiting M dwarfs: Conditions for atmospheric collapse and the implications for habitability. Icarus, 129, 450-465, doi:10.1006/ icar.1997.5793.

Kostiuk, T., K. E. Fast, T. A. Livengood, T. Hewagama, J. J. Goldstein, F. Espenak, and D. Buhl, 2001: Direct measurement of winds on Titan. Geophys. Res. Lett., 28, 2361-2364, doi:10.1029/2000GL012617.

Kraucunas, I., and D. L. Hartmann, 2005: Equatorial superrotation and the factors controlling the zonal-mean zonal winds in the tropical upper troposphere. J. Atmos. Sci., 62, 371-389, doi:10.1175/JAS-3365.1.

Kuang, Z., P. N. Blossey, and C. S. Bretherton, 2005: A new approach for $3 \mathrm{D}$ cloud-resolving simulations of large-scale atmospheric circulation. Geophys. Res. Lett., 32, L02809, doi:10.1029/2004GL021024.

Lee, S., 1999: Why are the climatological zonal winds easterly in the equatorial upper troposphere? J. Atmos. Sci., 56, 1353-1363, doi:10.1175/1520-0469(1999)056<1353: WATCZW $>2.0 . \mathrm{CO} ; 2$.

Lindzen, R. S., 1988: Instability of plane parallel shear flow (toward a mechanistic picture of how it works). Pure Appl. Geophys., 126, 103-121, doi:10.1007/BF00876917.

—_, and A. Y. Hou, 1988: Hadley circulations for zonally averaged heating centered off the equator. J. Atmos. Sci., 45, 2416-2427, doi:10.1175/1520-0469(1988)045<2416: $\mathrm{HCFZAH}>2.0 . \mathrm{CO} ; 2$.

Liu, J., and T. Schneider, 2010: Mechanisms of jet formation on the giant planets. J. Atmos. Sci., 67, 3652-3672, doi:10.1175/ 2010JAS3492.1.

$\longrightarrow$, and - 2011: Convective generation of equatorial superrotation in planetary atmospheres. J. Atmos. Sci., 68, 27422756, doi:10.1175/JAS-D-10-05013.1.

Markiewicz, W. J., and Coauthors, 2007: Morphology and dynamics of the upper cloud layer of Venus. Nature, 450, 633636, doi:10.1038/nature06320.

Matsuno, T., 1966: Quasi-geostrophic motions in the equatorial area. J. Meteor. Soc. Japan, 44, 25-42.

Merlis, T. M., and T. Schneider, 2010: Atmospheric dynamics of Earth-like tidally locked aquaplanets. J. Adv. Model. Earth Sys., 2, 13, doi:10.3894/JAMES.2010.2.13.

- , and - 2011: Changes in zonal surface temperature gradients and Walker circulations in a wide range of climates. J. Climate, 24, 4757-4768, doi:10.1175/2011JCLI4042.1.

Mitchell, J. L., and G. K. Vallis, 2010: The transition to superrotation in terrestrial atmospheres. J. Geophys. Res., 115, E12008, doi:10.1029/2010JE003587.

,,-- and S. F. Potter, 2014: Effects of the seasonal cycle on superrotation in planetary atmospheres. Astrophys. J., 787, 23, doi:10.1088/0004-637X/787/1/23.

O'Gorman, P. A., and T. Schneider, 2007: Recovery of atmospheric flow statistics in a general circulation model without nonlinear eddy-eddy interactions. Geophys. Res. Lett., 34, L22801, doi:10.1029/2007GL031779.

Pierrehumbert, R. T., 2011: A palette of climates for Gliese 581g. Astrophys. J., 726, L8, doi:10.1088/2041-8205/726/1/L8.

Pinto, J. R. D., and J. L. Mitchell, 2014: Atmospheric superrotation in an idealized GCM: Parameter dependence of the eddy response. Icarus, 238, 93-109, doi:10.1016/ j.icarus.2014.04.036.
Porco, C. C., and Coauthors, 2003: Cassini imaging of Jupiter's atmosphere, satellites, and rings. Science, 299, 1541-1547, doi:10.1126/science.1079462.

Potter, S. F., G. K. Vallis, and J. L. Mitchell, 2014: Spontaneous superrotation and the role of Kelvin waves in an idealized dry GCM. J. Atmos. Sci., 71, 596-614, doi:10.1175/ JAS-D-13-0150.1.

Randel, W. J., and I. M. Held, 1991: Phase speed spectra of transient eddy fluxes and critical layer absorption. J. Atmos. Sci., 48, 688-697, doi:10.1175/1520-0469(1991)048<0688: PSSOTE $>2.0 . \mathrm{CO} ; 2$.

Sánchez-Lavega, A., R. Hueso, and S. Pérez-Hoyos, 2007: The three-dimensional structure of Saturn's equatorial jet at cloud level. Icarus, 187, 510-519, doi:10.1016/ j.icarus.2006.10.022.

Saravanan, R., 1993: Equatorial superrotation and maintenance of the general circulation in two-level models. J. Atmos. Sci., 50, 1211-1227, doi:10.1175/1520-0469(1993)050<1211: ESAMOT $>2.0 . \mathrm{CO} ; 2$.

Sardeshmukh, P. D., and B. J. Hoskins, 1988: The generation of global rotational flow by steady idealized tropical divergence. J. Atmos. Sci., 45, 1228-1251, doi:10.1175/1520-0469(1988)045<1228: TGOGRF $>2.0 . \mathrm{CO} ; 2$.

Schneider, E. K., 1981: On the amplitudes reached by baroclinically unstable disturbances. J. Atmos. Sci., 38, 2142-2149, doi:10.1175/1520-0469(1981)038<2142: OTARBB $>2.0 . \mathrm{CO} ; 2$

Schneider, T., 2004: The tropopause and the thermal stratification in the extratropics of a dry atmosphere. J. Atmos. Sci., 61, 1317-1340, doi:10.1175/1520-0469(2004)061<1317: TTATTS $>2.0 . \mathrm{CO} ; 2$.

2006: The general circulation of the atmosphere. Annu. Rev. Earth Planet. Sci., 34, 655-688, doi:10.1146/ annurev.earth.34.031405.125144.

, and J. Liu, 2009: Formation of jets and equatorial superrotation on Jupiter. J. Atmos. Sci., 66, 579-601, doi:10.1175/ 2008JAS2798.1.

, and C. C. Walker, 2006: Self-organization of atmospheric macroturbulence into critical states of weak nonlinear eddyeddy interactions. J. Atmos. Sci., 63, 1569-1586, doi:10.1175/ JAS3699.1.

, and - 2008: Scaling laws and regime transitions of macroturbulence in dry atmospheres. J. Atmos. Sci., 65, 21532173, doi:10.1175/2007JAS2616.1

Schubert, G., 1983: General circulation and the dynamical state of the Venus atmosphere. Venus, D. M. Hunten et al., Eds., The University of Arizona Press, 681-765.

Shell, K. M., and I. M. Held, 2004: Abrupt transition to strong superrotation in an axisymmetric model of the upper troposphere. J. Atmos. Sci., 61, 2928-2935, doi:10.1175/JAS-3312.1.

Smith, K., G. Boccaletti, C. Henning, I. Marinov, C. Tam, I. Held, and G. Vallis, 2002: Turbulent diffusion in the geostrophic inverse cascade. J. Fluid Mech., 469, 13-48, doi:10.1017/ S0022112002001763.

Sobel, A. H., J. Nilsson, and L. M. Polvani, 2001: The weak temperature gradient approximation and balanced tropical moisture waves. J. Atmos. Sci., 58, 3650-3665, doi:10.1175/ 1520-0469(2001)058<3650:TWTGAA > 2.0.CO;2.

Stewartson, K., 1977: The evolution of the critical layer of a Rossby wave. Geophys. Astrophys. Fluid Dyn., 9, 185-200, doi:10.1080/03091927708242326.

Suarez, M. J., and D. G. Duffy, 1992: Terrestrial superrotation: A bifurcation of the general circulation. J. Atmos. 
Sci., 49, 1541-1554, doi:10.1175/1520-0469(1992)049<1541: TSABOT $>2.0 . \mathrm{CO} ; 2$.

Thuburn, J., and G. C. Craig, 2000: Stratospheric influence on tropopause height: The radiative constraint. J. Atmos. Sci., 57, 17-28, doi:10.1175/1520-0469(2000)057<0017: SIOTHT $>2.0$.CO;2.

Walker, C. C., and T. Schneider, 2006: Eddy influences on Hadley circulations: Simulations with an idealized GCM. J. Atmos. Sci., 63, 3333-3350, doi:10.1175/JAS3821.1.
Wang, P., and J. L. Mitchell, 2014: Planetary ageostrophic instability leads to superrotation. Geophys. Res. Lett., 41, 41184126, doi:10.1002/2014GL060345.

Williams, G. P., 2003: Barotropic instability and equatorial superrotation. J. Atmos. Sci., 60, 2136-2152, doi:10.1175/ 1520-0469(2003)060<2136:BIAES >2.0.CO;2.

Yamamoto, M., and M. Takahashi, 2006: Superrotation maintained by meridional circulation and waves in a Venus-like AGCM. J. Atmos. Sci., 63, 3296-3314, doi:10.1175/JAS3859.1. 